\title{
Fault-tolerant pancyclicity of augmented cubes
}

\author{
Wei-Wei Wang ${ }^{\mathrm{a}}$, Mei-Jie Ma ${ }^{\mathrm{b}}$, Jun-Ming $\mathrm{Xu}^{\mathrm{a}, *}$ \\ a Department of Mathematics, University of Science and Technology of China, Hefei 230026, China \\ ${ }^{\mathrm{b}}$ School of Mathematics and System Science, Shandong University, Jinan 250100, China \\ Received 26 December 2006; received in revised form 9 February 2007 \\ Available online 28 February 2007 \\ Communicated by L. Boasson
}

\begin{abstract}
As an enhancement on the hypercube $Q_{n}$, the augmented cube $A Q_{n}$, prosed by Choudum and Sunitha [S.A. Choudum, V. Sunitha, Augmented cubes, Networks 40 (2) (2002) 71-84], not only retains some favorable properties of $Q_{n}$ but also possesses some embedding properties that $Q_{n}$ does not. For example, $A Q_{n}$ is pancyclic, that is, $A Q_{n}$ contains cycles of arbitrary length for $n \geqslant 2$. This paper shows that $A Q_{n}$ remains pancyclic provided faulty vertices and/or edges do not exceed $2 n-3$ and $n \geqslant 4$. (c) 2007 Elsevier B.V. All rights reserved.
\end{abstract}

Keywords: Combinatorics; Networks; Augmented cubes; Pancycle; Fault tolerance

\section{Introduction}

It is well known that interconnection networks play an important role in parallel computing/communication systems. One of the central issues in evaluating a network is to study the embedding problem [14]. When a network is modeled by a graph, the embedding problem asks if a guest graph is a subgraph of a host graph, and an important benefit of graph embedding is that we can apply existing algorithms for guest graphs to host graphs. This problem has attracted a burst of studies in recent years. Cycle networks are suitable for designing simple algorithms with low communication cost. Since some parallel applications, such as those in image and signal processing, are originally designated on a cycle

\footnotetext{
The work was supported by NNSF of China (No. 10671191).

* Corresponding author.

E-mail address: xujm@ustc.edu.cn (J.-M. Xu).
}

architecture, it is important to have effective cycle embedding in a network.

A graph is pancyclic if it contains cycles of every length from its girth to order inclusive. A graph is of pancyclicity if it is pancyclic. The pancyclicity of many networks has been investigated in the literature (see, for example, $[1,4,5,9,7,16,18,19])$.

Edge and/or vertex failures are inevitable when a large parallel computer system is put in use. Therefore, the fault-tolerant capacity of a network is a critical issue in parallel computing. A graph $G=(V, E)$ is $k$ (resp. $k$-edge)-fault-tolerant pancyclic if $G-F$ is still pancyclic for any $F \subset E(G) \cup V(G)$ (resp. $F \subset E(G)$ ) with $|F| \leqslant k$. Fault-tolerant pancyclicity has been widely studied in many networks, such as $[2$, $4-8,10,13,15,17]$.

The hypercube network $Q_{n}$ has proved to be one of the most popular interconnection networks $[11,14]$. As a variant of $Q_{n}$, Choudum and Sunitha [3] introduced the augmented cube $A Q_{n}$ and proved $A Q_{n}$ is pancyclic. Re- 
cently, Ma et al. [12] improved this result by showing that $A Q_{n}$ is $(2 n-3)$-edge-fault-tolerant pancyclic for any $n \geqslant 2$. For the hybrid presence of edge and vertex failures, Hsu et al. [8] showed that $A Q_{n}$ is $(2 n-3)$ Hamiltonian for $n \geqslant 4$. In this paper, we improve the above-mentioned result by showing the following theorem.

Theorem. $A Q_{n}$ is $(2 n-3)$-fault-tolerant pancyclic for $n \geqslant 4$.

The proof of the Theorem is in Section 3. Section 2 gives the definition of the augmented cube $A Q_{n}$.

\section{Structure of augmented cubes}

The $n$-dimensional augmented cube $A Q_{n}(n \geqslant 1)$ can be defined recursively as follows: $A Q_{1}$ is a complete graph $K_{2}$ with the vertex set $\{0,1\}$. For $n \geqslant 2, A Q_{n}$ is obtained by taking two copies of the augmented cube $A Q_{n-1}$, denoted by $A Q_{n-1}^{0}$ and $A Q_{n-1}^{1}$, and adding $2^{n}$ edges between the two copies as follows.

Let $V\left(A Q_{n-1}^{0}\right)=\left\{0 u_{n-1} \ldots u_{2} u_{1}: u_{i}=0\right.$ or 1$\}$ and $V\left(A Q_{n-1}^{1}\right)=\left\{1 u_{n-1} \ldots u_{2} u_{1}: u_{i}=0\right.$ or 1$\}$. A vertex $u=0 u_{n-1} \ldots u_{2} u_{1}$ of $A Q_{n-1}^{0}$ is joined to a vertex $v=$ $1 v_{n-1} \ldots v_{2} v_{1}$ of $A Q_{n-1}^{1}$ if and only if either

(i) $u_{i}=v_{i}$ for $1 \leqslant i \leqslant n-1$; in this case, setting $v=$ $u^{h}$ or $u=v^{h}$, or

(ii) $u_{i}=\bar{v}_{i}$ for $1 \leqslant i \leqslant n-1$; in this case, setting $v=$ $u^{c}$ or $u=v^{c}$.

The augmented cubes $A Q_{1}, A Q_{2}$, and $A Q_{3}$ are shown in Fig. 1. It is proved in [3] that $A Q_{n}$ is a vertex transitive, $(2 n-1)$-regular, and $(2 n-1)$-connected graph with $2^{n}$ vertices for any positive integer $n$.

We call $u^{h}$ and $u^{c}$ the out-neighbors of $u$ and call the edges between $L$ and $R$ crossed edges, denoted by $E_{c}$. Clearly every vertex $u$ in $A Q_{n}$ is incident with two edges $\left(u, u^{h}\right)$ and $\left(u, u^{c}\right)$ in $E_{c}$. According to the above definition, we write this recursive construction of $A Q_{n}$ symbolically as $A Q_{n}=L \oplus R$, where $L \cong A Q_{n-1}^{0}$ and $R \cong$
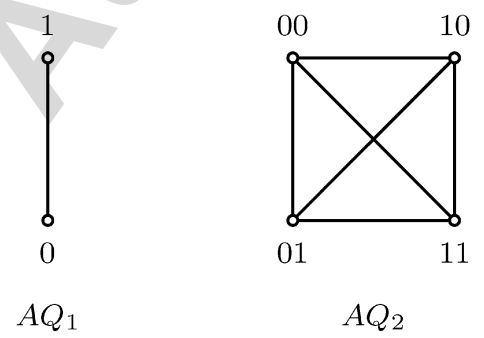

Fig. 1. Three augmented cubes $A Q_{1}, A Q_{2}$, and $A Q_{3}$.

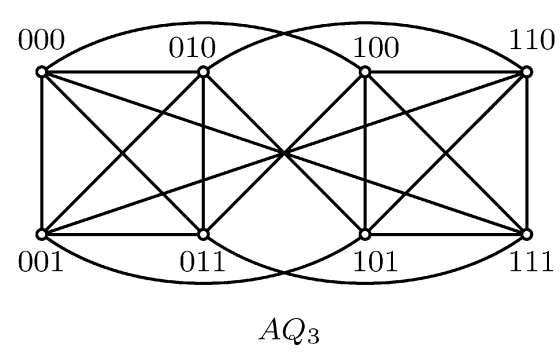

$A Q_{n-1}^{1}$. It is also clear that for two distinct vertices $u$ and $v$ in $A Q_{n}$ their out-neighbors $u^{h} \neq v^{h}$ and $u^{c} \neq v^{c}$. Moreover, for any two distinct vertices $u$ and $v$ in $A Q_{n-1}^{0}$ with $n \geqslant 4$, the four vertices $u^{h}, u^{c}, v^{h}$ and $v^{c}$ in $A Q_{n-1}^{1}$ are all distinct provided that $(u, v) \notin\{(u, v) \notin$ $E\left(A Q_{n-1}^{0}\right): u=0 u_{n-1} \ldots u_{1}$ and $\left.v=0 \bar{u}_{n-1} \ldots \bar{u}_{1}\right\}$.

\section{Proof of Theorem}

In this section, we give the proof of the Theorem stated in Introduction. For all the terminology and notation not defined here, we follow [14]. A graph $G$ is Hamiltonian connected if there is a Hamiltonian path between any two vertices of $G$, and is $k$-fault-tolerant Hamiltonian connected if $G-F$ remains Hamiltonian connected for any $F \subset E(G) \cup V(G)$ with $|F| \leqslant k$. The following two lemmas, due to Hsu et al. [8], are used in our proofs.

Lemma 1. $A Q_{n}$ is $(2 n-4)$-Hamiltonian connected for $n \geqslant 4$.

Lemma 2. For any four distinct vertices $u, v, x, y$ in $A Q_{n}(n \geqslant 2)$, there exist two disjoint ux-path $P_{1}$ and vy-path $P_{2}$ such that $P_{1} \cup P_{2}$ contains all vertices of $A Q_{n}$.

Proof of Theorem. We prove the theorem by induction on $n \geqslant 4$. For $n=4$, we have verified this conclusion with a computer by depth first search method within a polynomial time. Assume that the theorem is true for $A Q_{n-1}$ with $n \geqslant 5$. Let $F$ be any subset in $V\left(A Q_{n}\right) \cup$ $E\left(A Q_{n}\right)$ with $|F|=2 n-3, F_{v}=F \cap V\left(A Q_{n}\right), F_{e}=$ $F \cap E\left(A Q_{n}\right)$. We prove that there is a cycle of length $\ell$ in $A Q_{n}-F$ for every $\ell$ with $3 \leqslant \ell \leqslant 2^{n}-\left|F_{v}\right|$. To this end, let us denote $A Q_{n}=L \oplus R$, where $L \cong A Q_{n-1}^{0}$ and $R \cong$ $A Q_{n-1}^{1}, F^{L}=F \cap L, F^{R}=F \cap R, F_{v}^{L}=F_{v} \cap V(L)$, $F_{v}^{R}=F_{v} \cap V(R)$ and $F_{e}^{c}=F_{e} \cap E_{c}$.

Without loss of generality, we may assume $\left|F^{L}\right| \geqslant$ $\left|F^{R}\right|$. Then $\left|F^{R}\right| \leqslant n-2<2 n-6$ for $n \geqslant 5$, which implies that $R-F^{R}$ is Hamiltonian connected by 


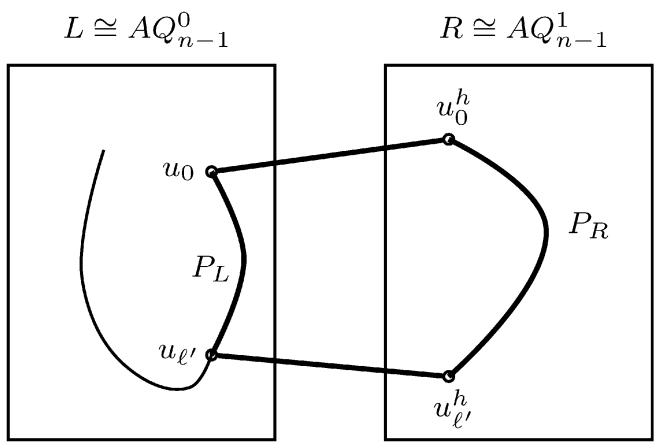

(a)

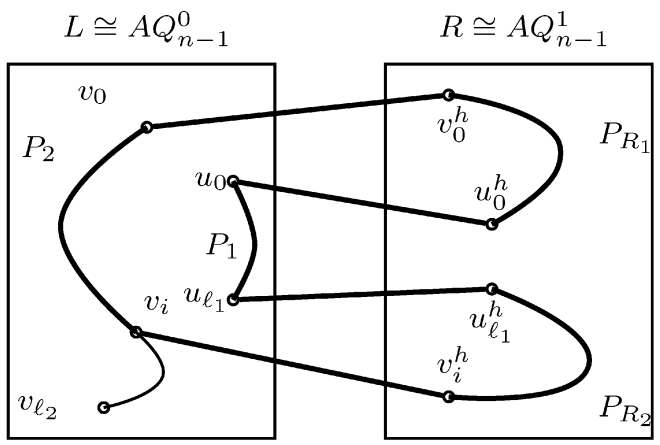

(c)

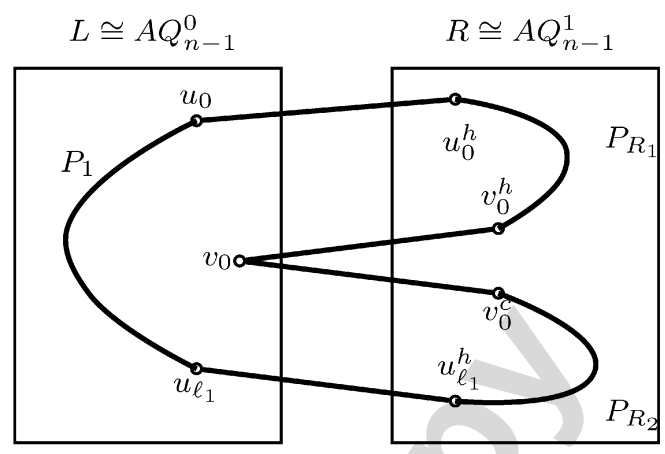

(b)

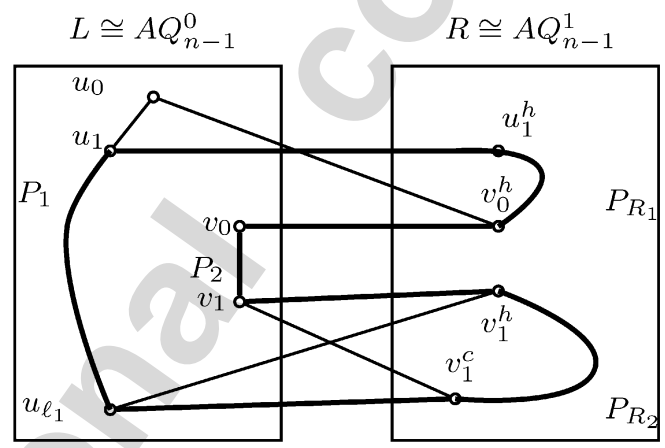

(d)

Fig. 2. Illustrations for the proof of Theorem. (A straight line represents an edge and a curve line represents a path between two vertices.)

Lemma 1. By the induction hypothesis, $R$ is $(2 n-5)$ fault-tolerant pancyclic, that is, for any $\ell$ with $3 \leqslant \ell \leqslant$ $2^{n-1}-\left|F_{v}^{R}\right|$, there is a cycle of length $\ell$ in $R-F^{R}$, and so in $A Q_{n}-F$.

Note $\left|F^{L}\right|+\left|F_{e}^{c}\right| \leqslant|F|=2 n-3<2^{n-1}$ for $n \geqslant 2$. There is a vertex $u$ in $L$ such that $\left\{\left(u, u^{h}\right),\left(u, u^{c}\right)\right\} \subset$ $E_{c} \backslash F_{e}^{c}$. Let $P_{R}$ be a $\left(u^{h}, u^{c}\right)$-path of length $2^{n-1}-$ $\left|F_{v}^{R}\right|-1$ in $R-F^{R}$ since $R-F^{R}$ is Hamiltonian connected. Then $\left(u, u^{h}\right) \cup P_{R} \cup\left(u^{c}, u\right)$ is a cycle of length $2^{n-1}-\left|F_{v}^{R}\right|+1$ in $A Q_{n}-F$.

Thus, to complete the proof of the theorem, we only need to prove that there is a cycle of length $\ell$ in $A Q_{n}-F$ for every $\ell$ with $2^{n-1}-\left|F_{v}^{R}\right|+2 \leqslant \ell \leqslant 2^{n}-\left|F_{v}\right|$. For a given $\ell$, let $\ell^{\prime}=\ell-2^{n-1}+\left|F_{v}^{R}\right|-1$. Then $1 \leqslant \ell^{\prime} \leqslant$ $2^{n-1}-\left|F_{v}^{L}\right|-1$. Consider the following two cases.

Case $1 .\left|F^{L}\right| \leqslant 2 n-4$.

We first claim that there is a $\left(u_{0}, u_{\ell^{\prime}}\right)$-path $P_{L}$ of length $\ell^{\prime}$ in $L-F^{L}$ such that at least one of the two sets of crossed edges $\left\{\left(u_{0}, u_{0}^{h}\right),\left(u_{\ell^{\prime}}, u_{\ell^{\prime}}^{h}\right)\right\}$ and $\left\{\left(u_{0}, u_{0}^{c}\right)\right.$, $\left.\left(u_{\ell^{\prime}}, u_{\ell^{\prime}}^{c}\right)\right\}$ is fault-free.

If $\left|F^{L}\right| \leqslant 2 n-5$, then $L-F^{L}$ contains a cycle $C$ of $2^{n-1}-\left|F_{v}^{L}\right|$ by the induction hypothesis. Thus there are $2^{n-1}-\left|F_{v}^{L}\right|$ distinct paths of length $\ell^{\prime}$ in $C$. Suppose to the contrary that there does not exist such a $\left(u_{0}, u_{\ell^{\prime}}\right)$ path $P_{L}$ in $L-F^{L}$. Then there are at least $2^{n-1}-\left|F_{v}^{L}\right|$ faults outside $L$, that is, $2 n-3=|F| \geqslant 2^{n-1}$ for $n \geqslant 5$, a contradiction.

If $\left|F^{L}\right|=2 n-4$, we claim that there is a Hamiltonian path $H$ in $L-F^{L}$. In fact, by the induction hypothesis, $L$ is $(2 n-5)$-Hamiltonian. Let $e$ be any element in $F^{L}$ and $C$ a Hamiltonian cycle in $L-\left\{F^{L}-\right.$ $\{e\}\}$. Then $e$ must be in $C$, and so $C-\{e\}$ is a Hamiltonian path in $L-F_{L}$.

Take any section of $H$ with length $\ell^{\prime}$ as the $\left(u_{0}, u_{\ell^{\prime}}\right)$ path $P_{L}$. Since $\left|F_{e}^{c}\right| \leqslant 1$, one of $\left\{\left(u_{0}, u_{0}^{h}\right),\left(u_{\ell^{\prime}}, u_{\ell^{\prime}}^{h}\right)\right\}$ and $\left\{\left(u_{0}, u_{0}^{c}\right),\left(u_{\ell^{\prime}}, u_{\ell^{\prime}}^{c}\right)\right\}$ is fault-free.

Thus, there exists a required $\left(u_{0}, u_{\ell^{\prime}}\right)$-path $P_{L}$ in $L-F^{L}$. Without loss of generality, assume $\left\{\left(u_{0}, u_{0}^{h}\right)\right.$, $\left.\left(u_{\ell^{\prime}}, u_{\ell^{\prime}}^{h}\right)\right\}$ is fault-free.

Let $P_{R}$ be a Hamiltonian $\left(u_{\ell^{\prime}}^{h}, u_{0}^{h}\right)$-path in $R-F^{R}$ since $R-F^{R}$ is Hamiltonian connected. Then $P_{L} \cup$ $\left(u_{\ell^{\prime}}, u_{\ell^{\prime}}^{h}\right) \cup P_{R} \cup\left(u_{0}^{h}, u_{0}\right)$ is a cycle in $A Q_{n}-F$ with length $\ell^{\prime}+2+\left(2^{n-1}-\left|F_{v}^{R}\right|-1\right)=\ell$ (see Fig. 2(a)).

Case 2. $\left|F^{L}\right|=2 n-3$.

In this case, all faults are in $L$, that is, $\left|F^{R}\right|=\left|F_{e}^{c}\right|$ $=0$ and $\ell^{\prime}=\ell-2^{n-1}-1$.

Suppose that $P_{L}$ is a $\left(u_{0}, u_{\ell^{\prime}}\right)$-path of length $\ell^{\prime}$ in $L-F^{L}$. Let $P_{R}$ be a $\left(u_{0}^{h}, u_{\ell^{\prime}}^{h}\right)$-path of length $2^{n-1}-1$ in $R$ since $R$ is Hamiltonian connected. Then $P_{L} \cup$ $\left(u_{\ell^{\prime}}, u_{\ell^{\prime}}^{h}\right) \cup P_{R} \cup\left(u_{0}^{h}, u_{0}\right)$ is a cycle in $A Q_{n}-F$ with length $\ell^{\prime}+2+\left(2^{n-1}-1\right)=\ell$ (see Fig. 2(a)). 
We now suppose that the length of any path in $L-$ $F^{L}$ is smaller than $\ell^{\prime}$. Noting that an edge $(x, y) \in F$ can be replaced by the vertex $x$ or the vertex $y$, we may, without loss of generality, assume $\left|F^{L}\right|=\left|F_{v}^{L}\right|=$ $2 n-3$.

Let $u, v$ be in $F_{v}^{L}$. By the induction hypothesis, there is a Hamiltonian cycle $C$ in $L-\left(F_{v}^{L} \backslash\{u, v\}\right)$. Then both $u$ and $v$ are in $C$ and not adjacent, otherwise $C-\{u, v\}$ is a path in $L-F_{v}^{L}$ with length $2^{n-1}-\left|F_{v}^{L}\right|-1 \geqslant \ell^{\prime}$, a contradiction. Let $P_{1}$ and $P_{2}$ be two sections of $C-\{u, v\}$. Then $P_{1}$ and $P_{2}$ are two disjoint paths in $L-F^{L}$. Denote the length of $P_{i}$ as $\ell_{i}$ for $i=1,2$. Then $\ell_{1}+\ell_{2}=2^{n-1}-\left|F_{v}\right|-2$. Let $P_{1}=\left(u_{0}, u_{1}, \ldots, u_{\ell_{1}}\right), P_{2}=\left(v_{0}, v_{1}, \ldots, v_{\ell_{2}}\right)$ and $C=\left(u, u_{0}\right) \cup P_{1} \cup\left(u_{\ell_{1}}, v\right) \cup\left(v, v_{\ell_{2}}\right) \cup P_{2} \cup\left(v_{0}, u\right)$. Without loss of generality, we may assume $\ell_{1} \geqslant \ell_{2}$. Then $\ell_{1}+1 \leqslant \ell^{\prime} \leqslant 2^{n-1}-\left|F_{v}\right|-1$.

Note that if $\left(u_{0}, v_{0}\right) \in E\left(A Q_{n}\right)$ then the path $P_{1} \cup$ $\left(u_{0}, v_{0}\right) \cup P_{2}$ has length $2^{n-1}-\left|F_{v}^{L}\right|-1 \geqslant \ell^{\prime}$, a contradiction, which implies $\left(u_{0}, v_{0}\right) \notin E\left(A Q_{n}\right)$. Similarly, $\left(u_{0}, v_{\ell_{2}}\right),\left(u_{\ell_{1}}, v_{0}\right),\left(u_{\ell_{1}}, v_{\ell_{2}}\right) \notin E\left(A Q_{n}\right)$. This fact implies that the four vertices $u_{0}^{h}, u_{0}^{c}, v_{0}^{h}$ and $v_{0}^{c}$ are all distinct, and so are $u_{\ell_{1}}^{h}, u_{\ell_{1}}^{c}, v_{\ell_{2}}^{h}$ and $v_{\ell_{2}}^{c}$.

(a) If $\ell_{2}=0$, then $P_{2}$ consists of a single vertex $v_{0}$. In this case, $\ell_{1}=2^{n-1}-\left|F_{v}\right|-2$, which implies $\ell^{\prime}=\ell_{1}+$ 1 and $\ell=2^{n}-\left|F_{v}\right|$. By Lemma 2, there are two disjoint $\left(u_{0}^{h}, v_{0}^{h}\right)$-path $P_{R_{1}}$ and $\left(v_{0}^{c}, u_{\ell_{1}}^{h}\right)$-path $P_{R_{2}}$ in $R$ such that $P_{R_{1}} \cup P_{R_{2}}$ contains all vertices in $R$. Then $\left(u_{0}, u_{0}^{h}\right) \cup$ $P_{R_{1}} \cup\left(v_{0}^{h}, v_{0}\right) \cup\left(v_{0}, v_{0}^{c}\right) \cup P_{R_{2}} \cup\left(u_{\ell_{1}}^{h}, u_{\ell_{1}}\right) \cup P_{1}$ is a cycle in $A Q_{n}-F$, of length $\ell_{1}+4+2^{n-1}-2=2^{n}-\left|F_{v}\right|=\ell$ (see Fig. 2(b)).

(b) If $\ell_{2} \geqslant 1$, then $\ell_{1} \leqslant 2^{n-1}-\left|F_{v}\right|-3$.

If $\ell_{1}+2 \leqslant \ell^{\prime} \leqslant 2^{n-1}-\left|F_{v}\right|-1$, then $2^{n-1}+\ell_{1}+3 \leqslant$ $\ell \leqslant 2^{n}-\left|F_{v}\right|$. Let $\ell^{\prime}=\ell_{1}+i+1$. Then $1 \leqslant i \leqslant \ell_{2}$ and $\ell=\ell_{1}+i+2^{n-1}+2$. Let $P_{2}^{\prime}$ be the section of $P_{2}$ from $v_{0}$ to $v_{i}$. Clearly, $u_{0}^{h}, u_{\ell_{1}}^{h}, v_{0}^{h}, v_{i}^{h}$ are four distinct vertices. By Lemma 2, there are two disjoint $\left(u_{0}^{h}, v_{0}^{h}\right)$ path $P_{R_{1}}$ and $\left(v_{i}^{h}, u_{\ell_{1}}^{h}\right)$-path $P_{R_{2}}$ such that $P_{R_{1}} \cup P_{R_{2}}$ contains all vertices of $R$. So, $\left(u_{0}, u_{0}^{h}\right) \cup P_{R_{1}} \cup\left(v_{0}^{h}, v_{0}\right) \cup$ $P_{2}^{\prime} \cup\left(v_{i}, v_{i}^{h}\right) \cup P_{R_{2}} \cup\left(u_{\ell_{1}}^{h}, u_{\ell_{1}}\right) \cup P_{1}$ is a cycle in $A Q_{n}$, of length $\ell_{1}+i+4+2^{n-1}-2=\ell_{1}+i+2^{n-1}+2=\ell$ (see Fig. 2(c)).

Now assume $\ell^{\prime}=\ell_{1}+1$. Then $\ell=2^{n-1}+\ell_{1}+2$.

If there is a vertex $v_{i}$ on $P_{2}$ whose two out-neighbors are different from out-neighbors of $u_{0}$ and $u_{\ell_{1}}$, then there are two disjoint $\left(u_{0}^{h}, v_{i}^{h}\right)$-path $P_{R_{1}}$ and $\left(v_{i}^{c}, u_{\ell_{1}}^{h}\right)$ path $P_{R_{2}}$ such that $P_{R_{1}} \cup P_{R_{2}}$ contains all vertices of $R$ by Lemma 2. So, $\left(u_{0}, u_{0}^{h}\right) \cup P_{R_{1}} \cup\left(v_{i}^{h}, v_{i}\right) \cup\left(v_{i}, v_{i}^{c}\right) \cup$ $P_{R_{2}} \cup\left(u_{\ell_{1}}^{h}, u_{\ell_{1}}\right) \cup P_{1}, u_{0}$ is a cycle in $A Q_{n}-F$, of length $2^{n-1}+\ell_{1}+2=\ell$.

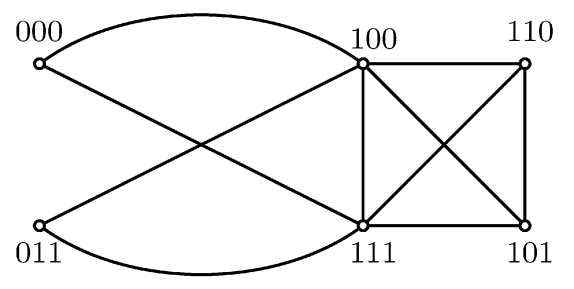

Fig. 3. $G=A Q_{3}-F$.

If the two out-neighbors of every vertex in $P_{2}$ are the same as the out-neighbors of $u_{0}$ or $u_{\ell_{1}}$, then $\ell_{2}=1$, that is, $P_{2}$ consists of a single edge $\left(v_{0}, v_{1}\right)$. Without loss of generality, assume $\left\{v_{0}^{h}, v_{0}^{c}\right\}=\left\{u_{0}^{h}, u_{0}^{c}\right\}$ and $\left\{v_{1}^{h}, v_{1}^{c}\right\}=$ $\left\{u_{\ell_{1}}^{h}, u_{\ell_{1}}^{c}\right\}$. Then $u_{1}^{h} \notin\left\{v_{0}^{h}, v_{0}^{c}, v_{1}^{h}, v_{1}^{c}\right\}$. By Lemma 2, there are disjoint $\left(u_{1}^{h}, v_{0}^{h}\right)$-path $P_{R_{1}}$ and $\left(v_{1}^{h}, v_{1}^{c}\right)$-path $P_{R_{2}}$ such that $P_{R_{1}} \cup P_{R_{2}}$ contains all vertices of $R$. Let $P_{1}^{\prime}$ be the section of $P_{1}$ from $u_{1}$ to $u_{\ell_{1}}$. Then $\left(u_{1}, u_{1}^{h}\right) \cup$ $P_{R_{1}} \cup\left(v_{0}^{h}, v_{0}\right) \cup P_{2} \cup\left(v_{1}, v_{1}^{h}\right) \cup P_{R_{2}} \cup\left(v_{1}^{c}, u_{\ell_{1}}\right) \cup P_{1}^{\prime}$ is a cycle in $A Q_{n}-F$, of length $2^{n-1}+\ell_{1}+2=\ell$ (see Fig. 2(d)).

The proof of the theorem is complete.

Remark. In $A Q_{3}$, let $F=\{001,010,(000,011)\}$, then $A Q_{3}-F$ is a graph $G$ shown in Fig 3. Clearly, there is no cycle of length 6 in $G$ since $G-\{100,111\}$ has three connected components. Therefore, $A Q_{3}$ is not 3-faulttolerant pancyclic. However, we can verify that $A Q_{3}$ is 2 -fault-tolerant pancyclic with a computer by depth first search method within a polynomial time.

\section{Conclusions}

As one of the most fundamental networks for parallel and distributed computation, cycles are suitable for developing simple algorithms with low communication cost. Edge and/or vertex failures are inevitable when a large parallel computer system is put in use. Therefore, the fault-tolerant capacity of a network is a critical issue in parallel computing. The fault-tolerant pancyclicity of an interconnection network is a measure of its capability of implementing ring-structured parallel algorithms in a communication-efficient fashion in the presence of faults. The augmented cube $A Q_{n}$, as a variation of the hypercube $Q_{n}$, not only retains some favorable properties of $Q_{n}$ but also possesses some embedding properties that $Q_{n}$ does not (see [3]). Choudum and Sunitha [3] showed that $A Q_{n}$ is pancyclic, that is, $A Q_{n}$ contains cycles of arbitrary length for $n \geqslant 2$. Ma et al. [12] showed that $A Q_{n}$ remains pancyclic provided faulty edges do not exceed $2 n-3$. For the hybrid presence of edge and vertex failures, Hsu et al. [8] showed that $A Q_{n}$ remains Hamiltonian provided faulty vertices 
and/or edges do not exceed $2 n-3$ and $n \geqslant 4$. In this paper, we improved these results by proving that $A Q_{n}$ remains pancyclic provided faulty vertices and/or edges do not exceed $2 n-3$ and $n \geqslant 4$.

In view of the fact that the hypercube network $Q_{n}$ is not pancyclic, $A Q_{n}$ is superior to $Q_{n}$ in terms of the fault-tolerant pancyclicity. This shows that, when the augmented cube is used to model the topological structure of a large-scale parallel processing system, our result implies that the system has larger capability of implementing ring-structured parallel algorithms in a communication-efficient fashion in the hybrid presence of edge and vertex failures than one of the hypercube network. Our further work is to determine fault-tolerant panconnectivity of $A Q_{n}$, that is, whether there is all paths of every length between any two distinct vertices in $A Q_{n}$ with failures.

\section{References}

[1] Y.A. Ashir, I.A. Stewart, On embedding cycles in $k$-ary $n$-cubes, Parallel Processing Letters 7 (1) (1997) 49-55.

[2] Y.A. Ashir, I.A. Stewart, Fault-tolerant embeddings of Hamiltonian circuits in $k$-ary $n$-cubes, SIAM Journal on Discrete Mathematics 15 (3) (2002) 317-328.

[3] S.A. Choudum, V. Sunitha, Augmented cubes, Networks 40 (2) (2002) 71-84.

[4] J. Fan, Hamilton-connectivity and cycle-embedding of Möbius cubes, Information Processing Letters 82 (2002) 113-117.

[5] S.-Y. Hsieh, N.-W. Chang, Pancyclicity on the Möbius cube with both faulty nodes and faulty edges, IEEE Transactions on Computers 55 (7) (2006) 854-863.

[6] S.Y. Hsieh, G.H. Chen, C.W. Ho, Fault-free Hamiltonian cycles in faulty arrangement graphs, IEEE Transactions on Parallel and Distributed Systems 10 (32) (1999) 223-237.
[7] S.-Y. Hsieh, C.-H. Chen, Pancyclicity on Möbius cubes with maximal edge faults, Parallel Computing 30 (2004) 407-421.

[8] H.-C. Hsu, L.-C. Chiang, J.J.M. Tan, L.-H. Hsu, Fault hamiltonicity of augmented cubes, Parallel Computing 31 (2005) 130145.

[9] W.-T. Huang, W.-K. Chen, C.-H. Chen, Pancyclicity of Möbius cubes, in: Proceedings of the Ninth International Conference on Parallel and Distributed Systems (ICPADS'02) on 17-20 Dec. 2002, pp. 591-596.

[10] W.-T. Huang, J.M. Tan, C.N. Hung, L.H. Hsu, Fault-tolerant hamiltonicity of twisted cubes, Journal of Parallel and Distributed Computing 62 (2002) 591-604.

[11] F.T. Leighton, Introduction to Parallel Algorithms and Architecture: Arrays, Trees, Hypercubes, Morgan Kaufmann, San Mateo, 1992.

[12] M.-J. Ma, G.-Z. Liu, J.-M. Xu, Panconnectivity and edgefault-tolerant pancyclicity of augmented cubes, Parallel Computing 33 (1) (2007) 36-42.

[13] Y.-C. Tseng, S.-H. Chang, J.-P. Sheu, Fault-free ring embedding in a star graph with both link and node failure, IEEE Transactions on Parallel and Distributed Systems 8 (30) (1997) 1185 1195

[14] J.-M. Xu, Topological Structure and Analysis of Interconnection Networks, Kluwer Academic Publishers, Dordrecht/Boston/ London, 2001.

[15] J.-M. Xu, Z.-Z. Du, M. Xu, Edge-fault-tolerant edge-bipancyclicity of hypercubes, Information Processing Letters 96 (4) (2005) 146-150.

[16] J.-M. Xu, M.-J. Ma, Cycles in folded hypercubes, Applied Mathematics Letters 19 (2) (2006) 140-145.

[17] J.-M. Xu, M.-J. Ma, Z.-Z. Du, Edge-fault-tolerant properties of hypercubes and folded hypercubes, Australasian Journal on Combinatorics 35 (2006) 7-16.

[18] M. Xu, J.-M. Xu, Edge-pancyclicity of Möbius cubes, Information Processing Letters 96 (4) (2005) 136-140.

[19] X. Yang, G.M. Megson, D.J. Evans, Locally twisted cubes are 4-pancyclic, Applied Mathematics Letters 17 (2004) 919925. 